\title{
Somatic mutations in haematopoietic cells contribute to atherosclerosis
}

\section{CHIP has}

a causal

role in the

progression of

atherosclerosis

and is a risk

factor for

coronary heart

disease
Clonal haematopoiesis of indeterminate potential (CHIP) is characterized by the expansion of haematopoietic clones that carry recurrent somatic mutations. CHIP has been previously linked with advanced age and increased risk of haematological cancer, but a new study published in The New England Journal of Medicine now reports that the presence of CHIP is associated with accelerated atherosclerosis in mice, and elevated risk of coronary heart disease in humans.

Advanced age is linked with an increased risk of cancer and cardiovascular disorders. "By mining previously generated exome sequencing data from people who did not have known blood cancer, we found that somatic mutations that cause clonal expansion of blood stem cells were relatively common in the elderly population," explains Siddhartha

\section{populion, explains Siddhartha}

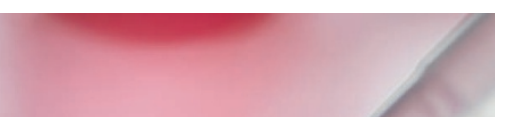

Jaiswal, lead author of the study.

"Those who had these mutations were ten times as likely to develop blood cancer in the future, and were also more likely to die of any cause." Further exploratory analysis revealed that this increased risk of death was attributable to cardiovascular causes, specifically coronary heart disease. The investigators sought to determine whether CHIP has a causal role in the development of atherosclerotic cardiovascular disease.

The investigators generated whole-exome sequencing data using blood samples obtained from four case-control studies (Biolmage, MDC, ATVB, and PROMIS) that together enrolled 4,726 patients with coronary heart disease and 3,529 healthy controls, to identify the presence of CHIP in peripheral blood cells. A fixed-effects meta-analysis combining data from the BioImage and MDC cohorts revealed that carriers of CHIP had a 1.9-fold increased risk of coronary heart disease compared with noncarriers (95\% CI 1.4-2.7, $P<0.001)$. Furthermore, a combined fixed-effects metaanalysis of the ATVB and PROMIS cohorts showed that CHIP was associated with a 4.0-fold increase in the risk of early-onset myocardial infarction in those aged $<50$ years (95\% CI 2.4-6.7, $P<0.001$ ). Mutations in ASXL1, DNMT3A, JAK2, or TET2 were individually linked with elevated risk of incident coronary heart disease. Furthermore, coronary artery calcification, a marker of atherosclerosis, was higher in carriers of CHIP than in noncarriers.

Having established an association between CHIP and coronary heart disease, the investigators sought to assess causality. Atherosclerosisprone, $L d l r$-knockout mice were transplanted with bone marrow from mice that lacked Tet2, the second most commonly mutated gene associated with clonal haematopoiesis. After 10 weeks receiving a high-fat, high-cholesterol diet, mice that received Tet2-deficient marrow had significantly greater aortic root lesion size compared with mice that had received control bone marrow. Deletion of Tet 2 was associated with excess production of inflammatory cytokines and chemokines.

Together, these observations indicate that CHIP has a causal role in the progression of atherosclerosis, and is a risk factor for coronary heart disease in humans. "We would next like to see if there are suitable interventions for those with CHIP to lower their risk of cardiovascular disease," proposes Jaiswal. "These findings might spur the development of new drugs that suppress CHIP clones or the inflammatory molecules that are overproduced."

Christie Ballantyne from Baylor College of Medicine in Houston, Texas, USA, who was not involved in the study, welcomes these novel findings. "This study identifies a new risk factor for atherosclerosis and might also help to explain why ageing is associated with increased heart disease and risk of leukaemia and myelodysplasia," he points out. "These findings also highlight the importance of leukocytes and inflammation in atherosclerosis in humans."

\section{Karina Huynh}

ORIGINAL ARTICLE Jaiswal, S. et al. Clonal hematopoiesis and risk of atherosclerotic cardiovascular disease. N. Engl.J. Med. http:// dx.doi.org/10.1056/NEJMoa1701719 (2017) 